\title{
Indicadores de monitoramento do câncer de colo de útero em um Município Maranhense
}

\author{
Monitoring indicators of cervical cancer in a maranhense municipal
}

\section{Indicadores de monitoreo del cáncer de colo de útero en un municipio maranhense}

Alayna de Araújo Rocha Souza; Maria Audenice Saldanha da Silva²; Francilene de Sousa Vieira ${ }^{2 *}$; Hele Sandra Barbosa Dias²; Márcia Regina Souza Batista²; Otacílio Tavares Fernandes²; Rafael Teles de Lima Cardoso²; Inecilda da Costa de Araújo; Maria Laura Sales da Silva Matos²; Sayonara Carvalho ${ }^{2}$

\section{RESUMO}

Objetivo: Analisar de forma descritiva o monitoramento do câncer de colo de útero no município de São Mateus do Maranhão. Métodos: Ressalta-se que a pesquisa se constitui de um estudo descritivo, de natureza quantitativa, com base me pesquisas realizadas na Coordenação do Programa de Atenção à Saúde da Mulher do Município de São Mateus do Maranhão -MA, bibliotecas digitais. Resultados: A maioria das mulheres que realizou o citopatológico possuía idades de 25 a 29 anos (160 mulheres), o que mostra uma ideia dentro do ideal e no ano de 2016 todas as faixas realizaram apenas um exame. Dos 1.157 laudos estudados, 928 mulheres estavam dentro da faixa etária recomendada, correspondendo a $80,2 \%$, percentual das amostras estudadas, concordante ao que é recomendado pelo Ministério da Saúde, com o alcance de $80 \%$ de cobertura. Houve também um percentual de amostras insatisfatórias com apenas 7 lâminas para leitura, equivalente a $0,60 \%$. Também houve lâminas satisfatórias que apresentaram como limitadas pois constatava ausência de células da endocérvice, dessecamento, presença de sangue, material escasso, e outros. Conclusão: Os achados da pesquisa evidenciam a necessidade de ações mais amplas em relação à realização do exame citopatológico na faixa etária recomendada pelo MS, mediante o percentual de exames realizados fora dessa faixa etária.

Palavras-Chave: Prevenção; Câncer; Colo do útero; Saúde Pública.

\section{ABSTRACT}

Objective: To describe, in a descriptive way, the cervical cancer screening in the municipality of São Mateus do Maranhão. Methods: The research is a quantitative descriptive study, based on research in the Program for the Coordination of Attention to Women's Health, São Mateus do Maranhão, MA, digital libraries. Results: Most of the women who did the cytopathology were aged 25 to 29 years (160 women), which shows an idea of the ideal and by 2016 all the bands just completed a test. Of the 1,157 awards studied, 928 women were within the recommended age range, corresponding to $80.2 \%$, a percentage of the samples studied, according to what is recommended by the Ministry of Health, with $80 \%$ coverage coverage. There was also a percentage of unsatisfactory samples with only 7 sheets for reading, equivalent to $0.60 \%$. There were also satisfactory leaves that were presented as limited because they found absence of endocervical cells, drying, presence of blood, scarce material and others. Conclusion: The research findings evidenced the need for broader actions regarding the accomplishment of the cytopathological examination in the age group recommended by the MS, by means of the percentage of exams performed outside this age group.

Keywords: Prevention; Cancer; Cervix; Public health.

${ }^{1}$ Hospital Geral de Peritoró, Núcleo de Estudos e Pesquisas.

${ }^{2}$ Hospital Geral de Peritoró. * E-mail: lennyenf93@gmail.com 


\section{RESUMEN}

Objetivo: analizar de forma descriptiva el monitoreo del cáncer de cuello de útero en el municipio de São Mateus do Maranhão. Métodos: Se resalta que la investigación se constituye de un estudio descriptivo, de naturaleza cuantitativa, con base a investigaciones realizadas en la Coordinación del Programa de Atención a la Salud de la Mujer del Municipio de São Mateus do Maranhão -MA, bibliotecas digitales. Resultados: La mayoría de las mujeres que realizaron el citopatológico tenían edades de 25 a 29 años (160 mujeres), lo que muestra una idea dentro del ideal y en el año 2016 todas las pistas sólo realizaron un examen. De los 1.157 laudos estudiados, 928 mujeres estaban dentro del grupo de edad recomendada, correspondiendo al $80,2 \%$, porcentaje de las muestras estudiadas, concordante a lo que es recomendado por el Ministerio de Salud, con el alcance del $80 \%$ de cobertura. También hubo un porcentaje de muestras insatisfactorias con sólo 7 láminas para lectura, equivalente al 0,60\%. También hubo láminas satisfactorias que presentaron como limitadas pues constataba ausencia de células de la endocérvice, desecamiento, presencia de sangre, material escaso, y otros. Conclusión: Los hallazgos de la investigación evidencian la necesidad de acciones más amplias en relación a la realización del examen citopatológico en la franja etaria recomendada por el MS, mediante el porcentaje de exámenes realizados fuera de esa franja etaria.

Palabras clave: Prevención; Cáncer; Colo del útero; Salud pública.

\section{INTRODUÇÃO}

No Brasil, o câncer do colo uterino representa um dos mais preocupantes problemas de saúde pública. Conforme o Instituto Nacional de Câncer (INCA), esse tipo de neoplasia apresenta-se a segunda mais prevalente no mundo e sua estimativa para o Brasil no ano de 2016 é de 16.340 casos novos (INCA, 2016).

O índice considerável de câncer do colo do útero faz com que seja classificado com o terceiro tipo de tumor mais prevalente na população feminina no Brasil, e em quarto lugar nas causas de mortes em mulher por câncer (INCA, 2017). A exposição das mulheres a fatores de risco e da eficiência dos programas de rastreamento são reflexos para as incidências encontradas na literatura. Em 99,7\% dos casos, o HPV (Papilomabírus Humano) tem relação ao câncer do colo de útero (FEITOSA; ALMEIDA, 2007).

Tendo em vista a importância desse tipo de câncer e sua repercussão na saúde pública, no ano de 1998 o Ministério da Saúde (MS) instituiu o Programa Nacional de Combate ao Câncer de Colo do Útero por meio da Portaria GM/MS no 3.040/98, (BRASIL, 2010). Após a transferência da coordenação do programa para o Instituto Nacional do Câncer (Portaria GM/MS no 788/99), o MS criou o Sistema de Informação do Câncer do Colo do Útero (Siscolo) como uma estratégia de monitoramento e gerenciamento das ações, constituindo um software para o armazenamento de dados sobre a identificação da paciente, informações demográficas, epidemiológicas e dos exames citopatológicos e histopatológicos realizados no Sistema Único de Saúde SUS (BRASIL, 2011).

O MS recomenda que os exames de rastreio comecem a partir dos 25 anos, após 2 exames normais com intervalo de 1 ano deve ser repetido a cada 3 anos até 64 anos. Segundo o que é preconizado pelo programa, que $80 \%$ dos exames para rastreio devem ser realizados na faixa etária com maiores casos da patologia (entre 25 e 64 anos de idade), afim de que o programa apresente maiores êxitos na redução da morbidade e nas taxas de cura.

A Secretaria do Estado da Saúde do Estado do Maranhão (SESMA) instituiu através do Programa saúde da mulher para o município de São Mateus do Maranhão o quantitativo de 1.631 exames de rasteio para o ano de 2016, estas metas foram estabelecidas foram estabelecidas de acordo com os dados do Instituto Brasileiro de Geografia e Estatísticas (IBGE) para a população feminina entre 25 a 64 anos está parametrizado em 0,47 e 0,40, respectivamente.

Com a finalidade de melhorar a cobertura do programa de prevenção do Câncer de colo de útero, foram estipulados indicadores com a finalidade de avaliar o alcance de metas e as estratégias do programa de 
enfrentamento do câncer de colo do útero em mulheres dentro da população alvo de 25 a 64 anos de idade. Este indicador tem sua importância, pois determina que $80 \%$ dos exames citopatológicos devem ser realizados nessa faixa, e também fornece parâmetros de avaliação do alcance do público desejado, por através de ações focadas na prevenção do câncer do colo do útero por meio do rastreamento precoce. (INCA, 2014).

Outro indicador importante consiste em medir a proporção de amostras insatisfatória em exame citopatológico do colo do útero, destacando que o percentual de amostras insatisfatória no total de exames realizados em um determinado local e período tem que ser menor que $5 \%$, este indicador está diretamente relacionado com a coleta do material e informa o percentual de amostras consideradas inadequadas 0 insuficiente para o diagnóstico, necessitando de repetição do exame, além de permitir avaliar e programar as ações de capacitações de recursos humanos, visando otimizar recursos e evitar perdas da adesão das mulheres para a realização do exame (INCA, 2014).

Considerando a importância do rastreamento do câncer de colo de útero, este estudo pretende realizar uma análise descritiva do monitoramento do câncer de colo de útero no município de São Mateus - MA, a partir da descrição dos indicadores de monitoramento do câncer de colo de útero no município de São Mateus do Maranhão - MA, da investigação da cobertura do câncer de colo de útero nas mulheres na faixa etária alvo de 25 a 64 anos que realizaram o exame de citopatológico no ano de 2016 e do percentual de amostras insatisfatórias.

\section{MÉTODOS}

Partiu-se das pesquisas realizadas na Coordenação do Programa de Atenção à Saúde da Mulher do Município de São Mateus do Maranhão -MA e em bibliotecas digitais. Em seguida procedeu-se a leitura do material levantado, elaborou-se um sumário provisório e rascunhos diversos e outras anotações. Apresentam-se os dados e informações sobre os resultados obtidos com os dados quantitativos serão transferidos e analisados no programa Microsoft Excel 2007.

Os percentuais obtidos no presente estudo serão dispostos em forma de gráficos, por meio de uma análise retrospectiva quantitativa dos dados de laudos dos exames cito patológicos coletados nas Unidades Básicas de Saúde (UBS) e cadastrados no Sistema de Informação do Câncer (SISCAN) do município de São Mateus do Maranhão, referente ao período de janeiro a dezembro de 2016. O cálculo dos indicadores seguirá as recomendações e formulas preconizadas pelo Ministério da Saúde.

\section{RESULTADOS E DISCUSSÃO}

No Brasil, o exame citopatológico é uma ferramenta estratégica de rastreamento para o câncer de colo do útero, recomendado para as mulheres com vida sexual ativa, prioritariamente de 25 a 64 anos de idade, visto que mulheres que não se enquadram nessa faixa etária apresentam menor incidência e mortalidade por essa neoplasia. Segundo o estudo conforme a distribuição de todos os exames realizados por faixas etárias observou-se que a maioria das mulheres que realizou o citopatológico possuía idades de 25 a 29 anos (160 mulheres), o que mostra uma ideia dentro do ideal.

Apesar das recomendações do MS, o presente estudo revelou um percentual expressivo de exames realizados fora dessa faixa etária. Dentro desse percentual observou-se que mulheres de 14 a 24 anos representaram 156 exames, apontando para o início precoce das relações sexuais, como um fator de risco para o desenvolvimento de câncer cervical, considerando que a puberdade e adolescência um período de vulnerabilidade, pois o epitélio cervical está em fase proliferativa e susceptível às alterações causadas por agentes transmitidos sexualmente, entre eles o HPV, aumentando o risco de desenvolver câncer cervical.

Todas as faixas realizaram apenas um exame durante o ano de 2016, mesmo aquelas mulheres que no laudo havia a sugestão para repetir o exame ou fazer acompanhamento com colposcopia e ginecologista (tabela 1). 
Tabela 1 - Número de exames realizados anualmente por faixa etária

\begin{tabular}{cc}
\hline Faixa etária & Exame \\
\hline 09 a 14 & 1 \\
15 a 24 & 1 \\
25 a 34 & 1 \\
35 a 44 & 1 \\
45 a 54 & 1 \\
55 a 64 & 1 \\
65 a 69 & 1 \\
$\geq 70$ & 1 \\
\hline
\end{tabular}

Fonte: SISCAN 2016.

Estudos apontam que o rastreamento precoce em mulheres em idade até 24 anos não tem impacto da redução da incidência e/ou mortalidade por câncer do colo do útero, porque a incidência é muito baixa e menos eficiente em relação a mulheres com faixa etária superior, já que a maioria dos achados dos diagnósticos apresenta-se já no estágio I, e nesse caso o rastreamento não é eficaz para detectá-los. Entretanto, o início precoce aumentaria os diagnósticos de manifestação citológica da infecção por HPV, esta considerada precursora para o CA de útero (BRASIL, 2013).

Uma recomendação estabelecida no Pacto pela vida, com o estabelecimento da ampliação do fornecimento do exame preventivo, com a finalidade de atingir $80 \%$ da população-alvo com idade entre $25 \mathrm{e}$ 64 anos (INCA, 2015).

Dos 1.157 laudos estudados, 928 mulheres estavam dentro da faixa preconizada pelo ministério, correspondendo à $80,2 \%$ (tabela 2). Com esse percentual da amostra estudada da população alvo para a prevenção do câncer de colo de útero, o município atingiu o percentual de cobertura para a faixa etária (gráfico 1). Esse indicador mostra a capacidade da rede de ofertar o exame, o que significa ainda uma boa cobertura.

Tabela 2 - Faixa etária das mulheres pesquisadas

\begin{tabular}{ccc|}
\hline Faixa etária & $\mathbf{N}$ & $\%$ \\
\hline 09 a 14 & 2 & 0,20 \\
15 a 24 & 156 & 13 \\
25 a 29 & 160 & 14 \\
30 a 34 & 148 & 13 \\
35 a 39 & 136 & 11 \\
40 a 44 & 117 & 10 \\
45 a 49 & 107 & 9 \\
50 a 54 & 112 & 10 \\
55 a 59 & 94 & 8 \\
60 a 64 & 54 & 5 \\
65 a 69 & 34 & 3 \\
$\geq 70$ & 37 & 3 \\
\hline TOTAL & $\mathbf{1 1 5 7}$ & $\mathbf{1 0 0}$ \\
\hline
\end{tabular}

Fonte: SISCAN, 2016. 
Gráfico 1 - Faixa etária alvo para prevenção do câncer de colo de útero

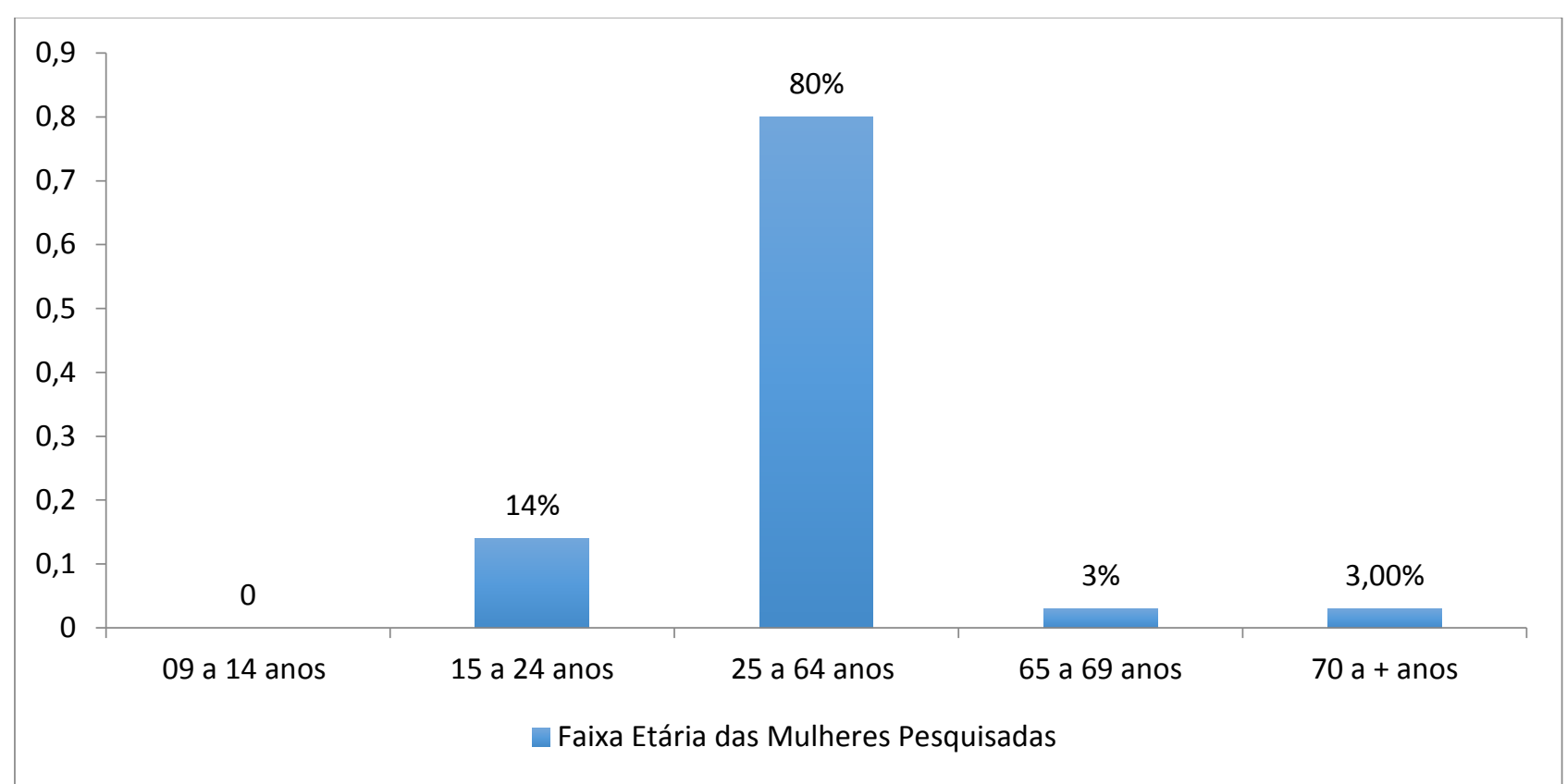

Fonte. SISCAN 2016.

Quanto ao percentual de amostras insatisfatória em exames citológicos estudos apontam que este não deve ser menor que $5 \%$ e que está diretamente relacionado com a qualidade da coleta. (INCA,2013). $O$ estudo apontou que o município está com este indicador satisfatório, atingiu $0.60 \%$ no índice de satisfação da qualidade da lamina, dos 1.157 laudos estudados apenas 7 apresentaram laminas insatisfatória para a leitura, por razões de dessecamento, material escasso, presença de sangue ou pus e espessamento no material coletada (gráfico 2).

Gráfico 2 - Demonstração do resultado quanto à amostra insatisfatória para o exame citológico.

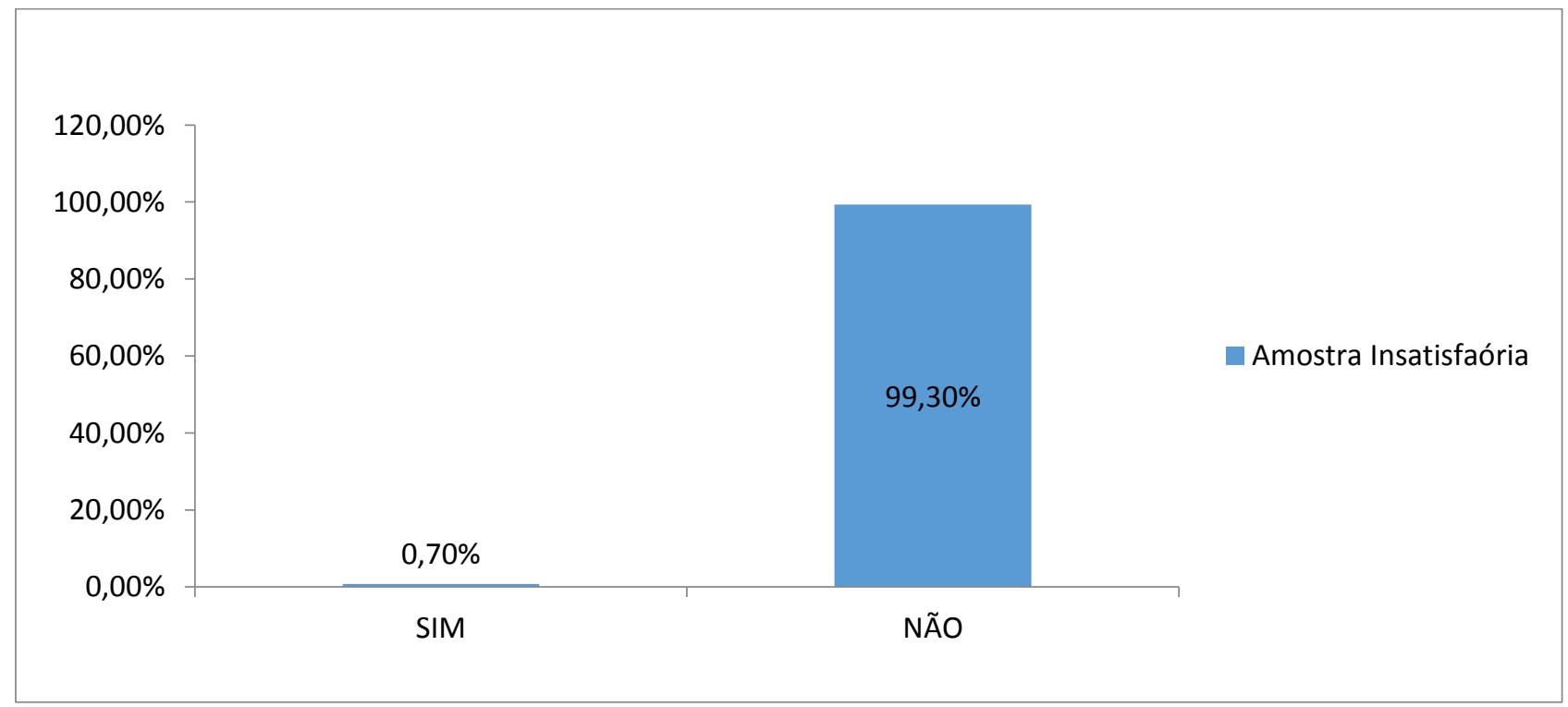

Fonte: SISCAN 2016 
É importante destacar que o estudo apontou um número muito elevado de laminas satisfatória (gráfico 3), porém limitadas por ausência de células da endocérvice, dessecamento, presença de sangue, material escasso, piócitos e atrofia celular, inviabilizando assim um resultado mais preciso. Segundo a nomenclatura uma amostra para ser considerada como insatisfatória enquadram-se nos seguintes requisitos: material acelular ou hipocelular ( $75 \%$ do esfregaço) por presença de sangue, piócitos, artefatos de dessecamento, contaminantes externos, intensa superposição celular e outros aspectos que inviabilizam a amostra (DIAS et al., 2010).

Gráfico 3 - Amostras satisfatórias, porém limitadas para a realização do exame citológico.

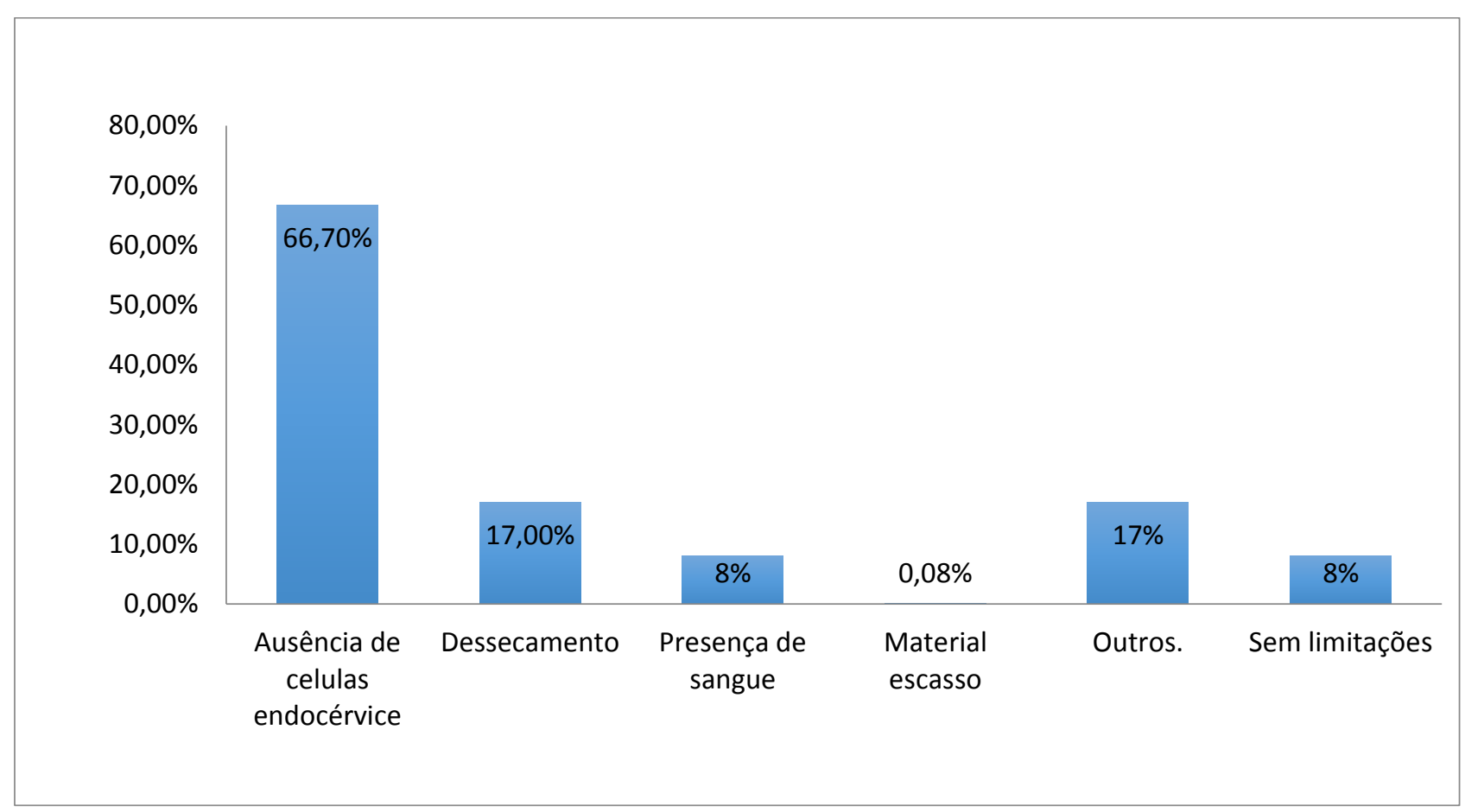

Fonte: SISCAN 2016.

\section{CONSIDERAÇÕES FINAIS}

Os achados da pesquisa evidenciam a necessidade de ações mais amplas em relação à realização do exame citopatológico na faixa etária recomendada pelo MS, mediante o percentual de exames realizados fora dessa faixa etária. Refere-se aos dados obtidos através de laudos dos exames cito patológicos coletados nas Unidades Básicas de Saúde (UBS) e cadastrados no SISCAN do município de São Mateus do Maranhão, referente ao período de janeiro a dezembro de 2016.

Essa discussão serviu para enriquecer acerca do rastreamento do câncer de colo de útero, e realizou-se também uma análise descritiva do monitoramento do câncer de colo de útero no município de São Mateus do Maranhão - MA.

A temática em estudo servirá para direcionar as ações envolvendo desde a estruturação do laboratório municipal até as coletas. Compreendemos que os resultados da pesquisa implicarão em tomadas decisões, como capacitação do profissional Enfermeiro para a coleta do material citológico, visto que houve um percentual de amostras insatisfatórias para a realização do exame, embora pequeno, mostrando a necessidade de avaliações mensais das laminas, com monitoramento e preparo das laminas com maior rigorosidade. 


\section{REFERÊNCIAS}

1. BRASIL. Ministério da Saúde. Instituto Nacional de Câncer (INCA). Sistema de informação do controle do câncer de mama (SISMAMA) e do câncer do útero (SISCOLO): manual gerencial. Rio de Janeiro: Inca; 2011.

2. CNES. Disponível

em: <http://cnes2.datasus.gov.br/Mod_Status_Carga_Arquivos.asp?nom_arq=CNES2111501710201711581020174010.BCK>. Acesso em: 9 out. 2017.

3. IBGE - Instituto Brasileiro de Geografia e Estatísticas. Censo Demográfico 2000 - Características Gerais da População. Disponível em: <http://www.censo2013.ibge.gov.br>. Acesso em: 9 out. 2017.

4. BRASIL. Informativo trimestral do Instituto Nacional de Câncer José Alencar Gomes da Silva (INCA). Ano 3 n. 3 , ago/dez. 2012.

5. BRASIL. Ministério da Saúde. Secretaria de Atenção à Saúde. Departamento de Atenção Básica. Controle dos cânceres do colo do útero e da mama. Brasília: Ministério da Saúde. 2. ed.

6. BRASIL. Brasília: Ministério da Saúde [periódico online]. 2013 [acesso em: 9 out. 2017]. Disponívelem://bvsms.saude.gov.br/bvs/publicacoes/controle_canceres_colo_utero_2013.pdf

7. BRASIL. Instituto Nacional do Câncer José Alencar Gomes da Silva. Coordenação Geral de Prevenção e Vigilância Divisão de Detecção Precoce e Apoio à Organização de Rede.Dez.2014. Acesso em 10 de Nov 2017.

8. BRASIL. Ministério da Saúde. Portaria n. 325, de 21 de fevereiro de 2008. Diretrizes Operacionais do Pactopela Saúde, Diário Oficial da União. Brasília. [periódicoonline2008. Disponívelem:http://dtr2001.saude.gov.br/sas/PORTARIAS/Port2008/GM/GM325.htm [Acesso em 09 de Out.]

9. BRASIL. Ministério da Saúde (MS). Instituto Nacional de Câncer (Inca). Programa Nacional de Controledo Câncer do Colo do Útero e Programa Nacional de Controle do Câncer de Mama. Rio de Janeiro: Inca; 2010.

10. DIAS M.B.K Et al. Rastreamento do câncer de colo do útero no Brasil: análise de dados do SISCOLO no período de 2002 a 2006. Epidemia Serviço Saúde 2010; 19(3):293-306.

11. FERREIRA, M. S. C. Et al. Valor do dilatador higroscópico para visualização do canal endocervical na conização com cirurgia de alta frequência. Revista Brasileira de Ginecologia. Obstetrícia, v. 26, n. 3, 2004.

12. FEITOSA T.M.P, Almeida RT. Perfil de produção do exame citopatológico para controle do câncer do colo do útero em Minas Gerais, Brasil, em 2002. Cad. Saúde Publica, v. 23, n.4, p.907-917, 2007.

13. INCA - Instituto Nacional de Câncer José Alencar Gomes da Silva. Coordenação de Prevenção e Vigilância. Divisão de Detecção Precoce e Apoio à Organização de Rede. - 2. ed. rev. atual. - Rio de Janeiro: INCA, 2016.

14. BRASIL. Falando sobre câncer de colo de Útero, 2. ed. rev. atual. - Rio de Janeiro: INCA, 2009.

15. BRASIL. Programa Nacional de Controle do Câncer do Colo do Útero e Programa Nacional de Controle do Câncer de Mama. Rio de Janeiro: Inca, 2017.

16. SISCAN - siscan.saude.gov.br//loguin.jsf. [acesso em nov de 2017].

17. SELLORS, J. W.; SANKARANARAYANAN, R. O exame colposcópico passo a apsso. In: Colposcopia e tratamento da neoplasia intraepitelial cervical: manual para principiantes. Lyon: IARC, 2004. cap. 5 . Disponível em: < https://screening.iarc.fr/doc/colpoptmanual.pdf>. .Acesso em: 07 out 2017. 